\title{
Applying a Process- and Dialogue-Centered Approach in Management Development at Loimaa Hospital
}

\author{
Ilkka Uusitaloa
}

\begin{abstract}
Management development has become a crucial element in the organizations function, and in the lives of their personnel, while working, learning, and developing have become more and more intimately interlinked. In a development-oriented working environment, there is a constant pressure to become "better", and this necessitates planned, strategic development programs. This paper examines the development of a process- and dialogue-centered approach and its implementation in a hospital environment. The production of information, learning, and professional expertise are seen as strongly dialogic and context-bound processes. During 2010-2011, a project to promote a patient-centered approach in nursing care and organizational reform was carried out at Loimaa Hospital, funded both from the Hospital's own resources and with the aid of a grant from the Finnish Work Environment Fund. The goals of the development process were: to reinforce the restructuring of services provision in the Hospital's departments; to support the spontaneously launched peer networking among charge nurses and assistant charge nurses; to model a patient-centered approach by means of mentoring; and to reinforce dialogic team working in pairs on the wards. This paper summarises the project's findings and describes the principles of the process-centered development.
\end{abstract}

\section{Keywords}

Process-centered development, dialogue, peer-groups, mentoring Loimaa Hospital (Loimaan aluesairaala)

Loimaa Hospital is one of two regional hospitals within the "Hospital District of Southwest Finland", and serves a population of around 85,000. In addition to the municipalities within its immediate official catchment area, it also provides some services for other adjacent municipalities which formally belong to different Hospital Districts (Satakunta, Kanta-Häme, and Pirkanmaa). There are almost 300 persons working at Loimaa Hospital: approximately 230 on the Hospital's own payroll, and the rest under some other divisions within the Hospital District. Since the beginning of 2009, Loimaa Hospital has been a member of the Hospital District's specialist medical care network, together with seven other hospitals in Salo, Uusikaupunki, and the region's main city, Turku (Uusitalo, Kosonen, and Isotalo 2011: 10).

Two of the goals set for the Hospital District's strategic plan for 2014-2016 were to establish parity of care and a clear division of labor between the different hospitals in the network, and to expedite

aTurku University of Applied Sciences, Finland

\section{Correspondent Author:}

Ilkka Uusitalo, Turku University of Applied Sciences, Ruiskatu 8, FI-20700 Turku, Finland 
access to care across the whole region. As part of this plan, in December 2015, the general wards and specialist in-patient wards in Loimaa and Uusikaupunki were combined into an integrated system. The strategic plan for 2017-2018 proposes to continue this process of integration among general medical care, specialist care, and welfare services, and the provision of equal levels of care across the region. At a meeting in Turku on February 29, 2016 to discuss the 2017-2018 strategic plan, four strategic goals were identified: patient-centeredness, parity of care, the well-being of the staff, and on-going improvement and renewal.

\section{GOALS OF THE DEVELOPMENT PROCESS}

During 2010-2011, a project to promote a patient-centered approach in nursing care and organizational reform, was carried out at Loimaa Hospital, funded both from the Hospital's own resources and with the aid of a grant from the Finnish Work Environment Fund. The goals of the development process were: to reinforce the restructuring of services provision in the Hospital's departments; to support the spontaneously launched peer networking among charge nurses and assistant charge nurses; to model a patient-centered approach by means of mentoring; and to reinforce dialogic team working in pairs on the wards.

In the planning group, the goals for the development project were formulated as follows: consolidation of the departmental reorganization within the Hospital, and development of medical care, by establishing communal learning structures, including peer networking for senior nursing staff (charge nurses) and a mentor-actor system for management and bedside nursing staff. An additional goal was to reinforce collaboration and mutual trust between lower management and the departments, and systematic thinking.

The long-term goal was to develop for all departments, on the basis of experience gained from the mentoring program, a shift-based duo-teamwork model to improve care. A systematic mentoring program has been shown to be an effective model for professional development and psychosocial support, and for promoting professional growth and improving skills among the nursing staff (Nikki, Koivunen, and Suominen 2010: 27).

\section{DEVELOPMENT RESEARCH: MATERIALS AND METHODS}

In the development project, especially in the peer meetings for charge nurses, a process-centered and dialogic approach was applied. In these peer-group meetings with an external expert also being present, the charge nurses and assistant charge nurses discussed topics which they themselves had proposed and which had been identified as currently relevant and important by a majority of senior management. In some cases, these meetings were supported by means of small-scale advance tasks. The role of the external expert was to provide advice about developmental methods which would maintain the process of development and promote contacts for dialogue between the everyday discourses used at different levels (Juuti 2006: 262).

The peer-group meetings lasted 90 minutes each, and the modus operandi consisted of the exchange of ideas and experiences, and the search for solutions applicable to the participants' own management tasks and to the shared challenges as the new departmental structure took effect. At the end of the project, in May 2011, participants were asked on a Webropol questionnaire to evaluate the significance and impact of the peer-group network project. Feedback was also collected about the experimental mentor-actor program, by means of open-ended questions put to the nurses and assistant nurses. Identical questions were put to both groups.

\section{DEVELOPMENT PROJECTS AS SUPPORT FOR STRATEGIC CHANGE}

Developmental projects are increasingly being 
recognized as a crucial element in organizational strategy, strategic thinking, and strategic management. Organization Development (OD) and Organization Transformation (OT) provide strategies for developing individuals within organizations. Both approaches are focused on individuals, personal relationships, processes, norms, organizational structures, and the improvement of organizational culture (Kondalkar 2009: 3).

In organizational development, there are many different features on which resources and effort may at different times be focused. Development almost always also requires recognition of the learning and skills needs of the organization, and the evaluation and choice of the most appropriate development methods. Heiskanen (2007: 257) suggested that needs for learning and development often emerge during or in anticipation of a situation of change. Predictive, proactive development is dependent on thorough familiarity with the current state of the organization, and a strategic perspective on how the organization should prepare for the imminent changes and challenges in its operational context.

The goal of development is to get certain aspect or aspects in the organization to function more smoothly or with a qualitatively better outcome, not forgetting improved productivity, which is particularly crucial for commercial enterprises. Without such outcomes, development has no point, and the resources spent on it are being wasted. Kesti (2005: 10) highlighted four human factors for success which impact most significantly on the organization's productivity and the personnel's work stamina: management, the organizational culture, skills, and internal communication.

\section{A PROCESS-CENTERED AND DIALOGIC FRAME OF REFERENCE FOR ORGANIZATIONAL DEVELOPMENT}

Among the most important prerequisites for successful process-centered and dialogic development and consultancy is thorough familiarity with the daily routines of the work community, and ensuring that the development process primarily takes place from the bottom up, is comprehensive in scope, and involves both individual and collective dialogue (see Figure 1). The focus needs to be on people's experiences, thoughts, and the meanings (interpretations) that they perceive.

The objective is for the working community to learn from and develop its own operations through collective evaluation, aiming at collaboratively established goals, and by doing things at work differently and better. This requires guidance and intervention by an outside consultant, to ensure that dialogue takes place, and that development is goal-oriented and change-driven (Murto 1995: 25-35).

Comprehensiveness in scope implies, in the author's view, three distinct concepts:

Firstly, it means that the work community, or the persons participating in the development process, need to explore collectively the vision, strategy, and goals of the organization. The patterns of interaction and collaboration between different units and levels of the hierarchy need to be examined, and people need to learn how to discuss these, so that they can gradually formulate shared and recognized meanings that they attach thereto;

Secondly, development is comprehensive in scope inasmuch as during development, attention is paid not only to the functioning of individuals' own unit, but of the organization as a whole;

Thirdly, development is comprehensive in scope inasmuch as it triggers in people contradictory reactions, which should not be ignored in the development process, but can in the best case be recognized as a valuable resource, and pointer ("silent signal") for the direction of development.

Positive feelings help to promote development, but people's negative feelings and expressions of feelings also need to be heard, and their origins explored. 


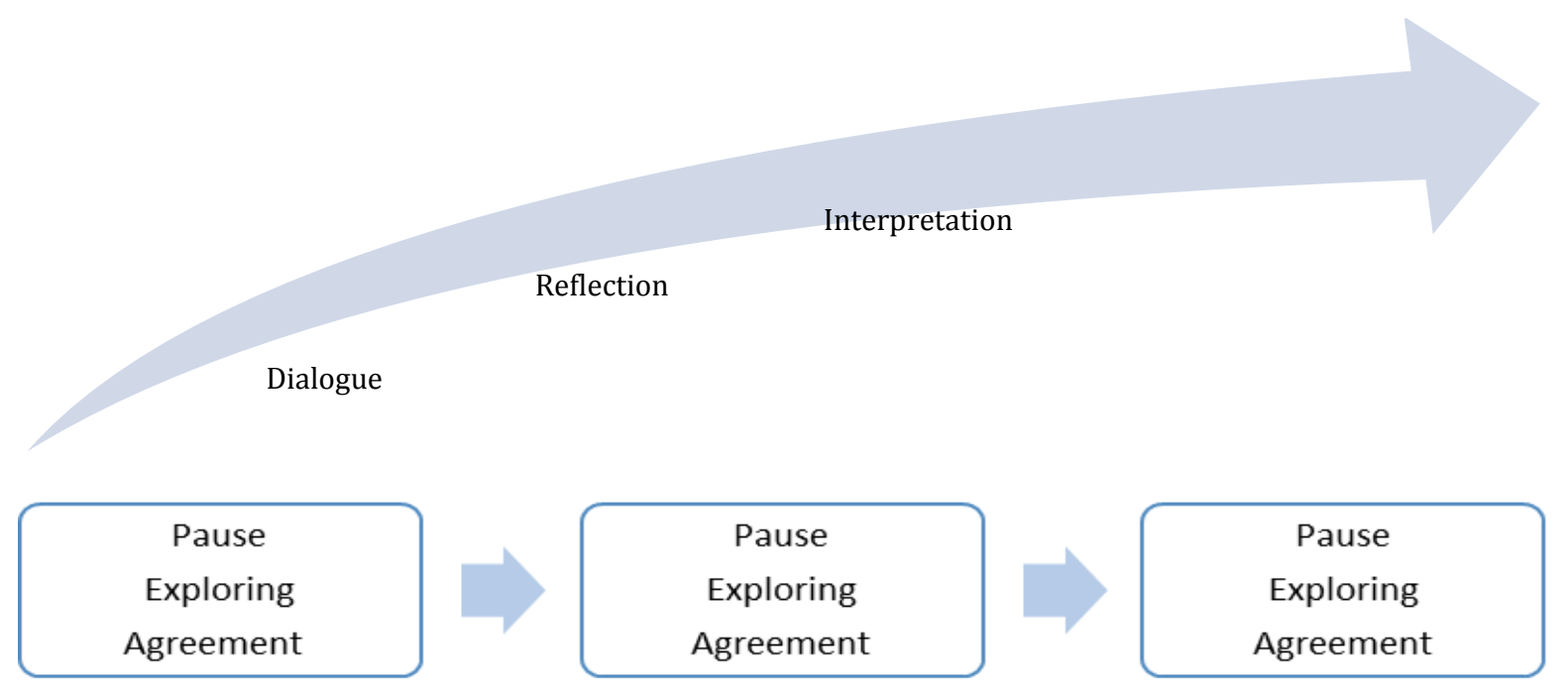

Work and activity in everyday operations (individuals' experiences)

Figure 1. Frame of Reference for Process-Centered and Dialogic Development [Applying the Model From Murto (1995)].

Often, negative comments and excessive criticism are a reflection of the fact that those involved in development perceive the development as in some way threatening their identity at work and in the work community.

Vartiainen (2005: 142) commented that each individual is at the center of their own world and their own situation, and will perceive life and issues from that perspective. When the consultant feels able to acknowledge criticism and negative reactions openly, and to spend time on the drafting of solutions, they may be able in this way to demonstrate to the work community the modus operandi which the community itself will later have need of.

The strengths of a process-centered and dialogic approach to development are above all that people can learn to discuss, in the generation of communal knowledge, to learn from each other, in reinforcing a culture of consensus, and at best to strengthen a sense of community. This does however presuppose in the consultant the ability to promote and maintain dialogue, and correspondingly, the ability to discard the traditional consultant's role as all-knowing expert. They need to be able to combine experiential and theoretical understanding in such a way that people can learn, and be aware of, new perspectives and even solutions for problems and challenges within the work community. Correspondingly, successful development is supported by the collective foregrounding and transformative examination of the work community's working processes, behavioral processes, and processes of change.

\section{OUTCOMES AND IMPACTS OF DEVELOPMENT}

Towards the end of the development process, a Webropol questionnaire was used to gather perceptions of its impact on the Hospital's operations, and the participants' experiences of the project. The findings indicated that peer-group networking had reinforced charge nurses' confidence in their management responsibilities. Six of the 12 respondents felt that participation had impacted 
positively on their awareness of skills within the work community and of superior-subordinate relationships. Eight of the charge nurses felt that their own interest in the Hospital's strategy and objectives had increased as a result of the networking. Ten of them considered that networking had improved interaction between charge nurses, communication between departments, and had increased levels of collaboration.

The mentor-actor discussions between line-management personnel and their immediate supervisors had been found useful, and the goals set for mentoring had been achieved. Even difficult topics had been successfully addressed. These "actors" felt that mentoring had provided a good means of learning. For nursing staff in general, however, the goals for the mentoring project had not been fully achieved. The charge nurses felt that motivating and encouraging nursing staff to participate by means of mentoring was a challenge. In principle, they saw the development of mentoring as a good method for developing staff morale, but developing it and establishing it as a standard procedure would place demands on departments' resources and would be a long-term project, since the results are not immediately to be seen (cf. Chao 1997: 24).

\section{CONCLUSIONS}

In process-centered and dialogic development and consultancy, it is essential to learn how to discuss people's experiences related to work, the organisation, the objectives of a person's role, practices, personal relationships, and development, among other things. All this will produce information and promote the change process as part of development. In the same way, different opinions, new insights, ideas, and suggested solutions can only be achieved through discussion and shared dialogue. Therefore, producing information, learning, and professional development are, by nature, social processes. The management has a great impact on the employee's ability to learn at work and on the development of a learning-focused workplace culture (Eraut 2007: 420).

\section{Note}

1. Varsinais-Suomen Sairaanhoitopiiri. Retrieved (http://www. vsshp.fi/).

\section{References}

Chao, G. T. 1997. "Mentoring Phases and Outcomes.” Journal of Vocational Behavior 51(1):15-28.

Eraut, M. 2007. "Learning From Other People in the Workplace." Oxford Review of Education 33(4):403-422.

Heiskanen, T. 2007. "The Spaces of Learning in the Information Society." Pp. 23-257 in Work, Identity and Learning, edited by A. Eteläpelto, K. Collin, and J. Saarinen. Helsinki: WSOY.

Juuti, P. 2006. Organisational Behaviour. Helsinki: Otava.

Kesti, M. 2005. Silent Signals-Key to Organisational Development. Helsinki: Edita.

Kondalkar, V. G. 2009. Organization Development. New Delhi: New Age International (P) Ltd., Publishers.

Murto, K. 1995. Process Management. Towards Process-Centred Development in the Workplace. 2nd ed. Jyväskylä: Jyväskylän Koulutuskeskus Oy.

Nikki, L., M. Koivunen, and T. Suominen. 2010. "Mentoring a New Employee.” Exploratory Nursing 3:20-29.

"The Strategy of the Hospital District of Southwest Finland 2017-2018." 2016. Discussion paper for meeting prior to Hospital District Council meeting. February 29, 2016, Turun T-sairaala, unpublished document.

Uusitalo, I., L. Kosonen, and A. Isotalo. 2011. "Empowered by Peer Support and Mentoring. Social Learning Structures Supporting Change in Loimaa Hospital." Reports 124. Turku University of Applied Sciences.

Vartiainen, E. 2005. "Understanding the Role of Dialogue in Consultancy." In Adult Education 2. Professional Development, pp. 141-146.

\section{Bio}

Ilkka Uusitalo, Licentiate of Philosophy, senior advisor in Turku University of Applied Sciences, Finland; research fields: professional learning and learning in the workplace. 